\title{
Coronavirus Disease (COVID- 19) Pandemic in Nigeria: A Review of the Basic Facts
}

\author{
Ya'aba Yakubu $^{1,2^{*}}$, Okposhi, Usman Shuaibu², Abdullahi Sani Ramalan ${ }^{2}$ \\ ${ }^{1}$ Department of Microbiology and Biotechnology, National Institute for Pharmaceutical Research and Development (NIPRD) Abuja, Nigeria \\ ${ }^{2}$ Department of Microbiology, Federal University of Lafia, Nasarawa State, Nigeria
}

DOI: $10.36348 /$ sjpm.2020.v05i08.007 $\quad$ | Received: 10.08 .2020 | Accepted: 18.08 .2020 | Published: 20.08 .2020

*Corresponding author: Ya'aba Yakubu

\section{Abstract}

Coronaviruses was first discovered in the late 1960s and were responsible for most respiratory and gastrointestinal infections in the human population. The present COVID-19 outbreak has affected over 210 countries including Nigeria. It is one of the largest respiratory disease affecting several countries simultaneously; a novel strain of Coronavirus (SARS$\mathrm{CoV}-2$ ) has been identified as the causative agent. Sequel to the advice of the International Health Regulation Emergency Committee, the Director-General of WHO declared that, COVID-19 outbreak has become a Public Health Emergency of International Concern (PHEIC) on $30^{\text {th }}$ January, 2020; and characterized it as a pandemic on $11^{\text {th }}$ March, 2020. The success in curtailing the pandemic (COVID-19) depends largely on better understanding of the basic facts. The aim of this review was to examine the basic facts such as, origin, spread, morphology, symptom, diagnosis, precautionary measures with WHO/NCDC/National guidelines, management and treatment, vaccine design and development, pathogenicity and ethical implications.

Keywords: Coronaviruses, COVID-19, Nigeria, Basic Facts, SARSCoV-2, WHO, NCDC, National Guidelines.

Copyright @ 2020: This is an open-access article distributed under the terms of the Creative Commons Attribution license which permits unrestricted use, distribution, and reproduction in any medium for non-commercial use (NonCommercial, or CC-BY-NC) provided the original author and sources are credited.

\section{INTRODUCTION}

\section{Origin, Morphology, Symptoms and Index case of COVID-19}

Coronaviruses was first discovered in the late 1960s and were responsible for most respiratory and gastrointestinal infections in the human population. The class of coronavirus was so-called for the reason that the virions have a distinctive characteristic of crownlike appearance under the electron microscope [1]. These viruses mainly affect the epithelial cells of the respiratory and gastrointestinal tracts, thereby causing numerous respiratory infections including pneumonia $[2]$.

There are two strains of the coronavirus family which include Severe Acute Respiratory Syndrome Coronavirus (SARS-CoV) and the Middle East Respiratory Syndrome Coronavirus (MERS CoV) that were accountable for preceding outbreaks worldwide. Severe Acute Respiratory Syndrome Coronavirus (SARS-CoV) was responsible for a global outbreak that started off in Guangdong Province, China in the years 2002 and 2003. The disease affected over an estimated eight thousand individuals causing severe pulmonary infections in many countries of the world [3]. Similarly, in 2012 an outbreak of MERS-COV infection was also first noticed in Saudi Arabia and was responsible for severe respiratory illnesses in some countries in the Middle East [4].

There was an outbreak of a new Coronavirus infection which caused acute respiratory syndrome of unknown aetiology was reported from China in the late December 2019. Utmost of the initial cases were linked to a seafood market (wet market) in Wuhan, Hubei Province [5]. The novel coronavirus 2019 is presently named severe acute respiratory syndrome coronavirus-2 (SARSCoV-2) while the illness associated with it is referred to as COVID-19 [6].

The early outbreak of COVID-19 was seemed to be a zoonotic transmission from bat to man and as the outbreak progresses, it was manifest that human to human transmission of the virus via close contact was also possible, particularly infections among health care workers [7]. The Severe Acute Respiratory Syndrome Coronavirus-2 (SARSCoV2) was identified to be responsible for severe cases of pneumonia in Wuhan. The full genome sequencing and phylogenic analysis showed that the SARS-CoV-2 is a $\beta$ eta $(\beta)$ Coronavirus in the same subgenus as the SARS virus but in a 
different clad. It was similar to a bat Coronavirus thereby suggesting that the primary source may be from bats $[8,9]$. The current Coronavirus Disease (COVID19) outbreak has affected over 250 countries including Nigeria [9].

However, the initial or first case of COVID-19 from China was informed to the World Health Organization (WHO) on the 31st of December 2019. The infection was observed to spread comparatively quickly to some other countries and by the end of January, 2020 precisely (30th January 2020), the WHO declared COVID-19 outbreak as a Public Health Emergency of International Concern (PHEIC) [10] and announced the new Coronavirus disease as COVID-19 (a shortening of COronaVIrus Disease-19) on the 11th
February 2020, [11] and thereafter, formally declared it a pandemic on 11th March 2020. The person to person spread of SARS-CoV-2 is primarily through close contact with an infected person and through respiratory droplets, saliva or discharges from the nose when an infected individual coughs or sneezes. The structure of coronavirus is shown in figure1 [12]. The spread of the virus is quite effective and as a consequence, the virus rapidly spreads from person to persons. There is strong indication that the virus can be transmitted by people who have a mild infection or those who are not showing symptoms (asymptomatic) of COVID-19. Also, an indirect spread has also been recognized to occur through touching contaminated objects and surfaces. The incubation period of COVID-19 ranges from 2 to 14 days but averages about 7 days $[12,13]$.

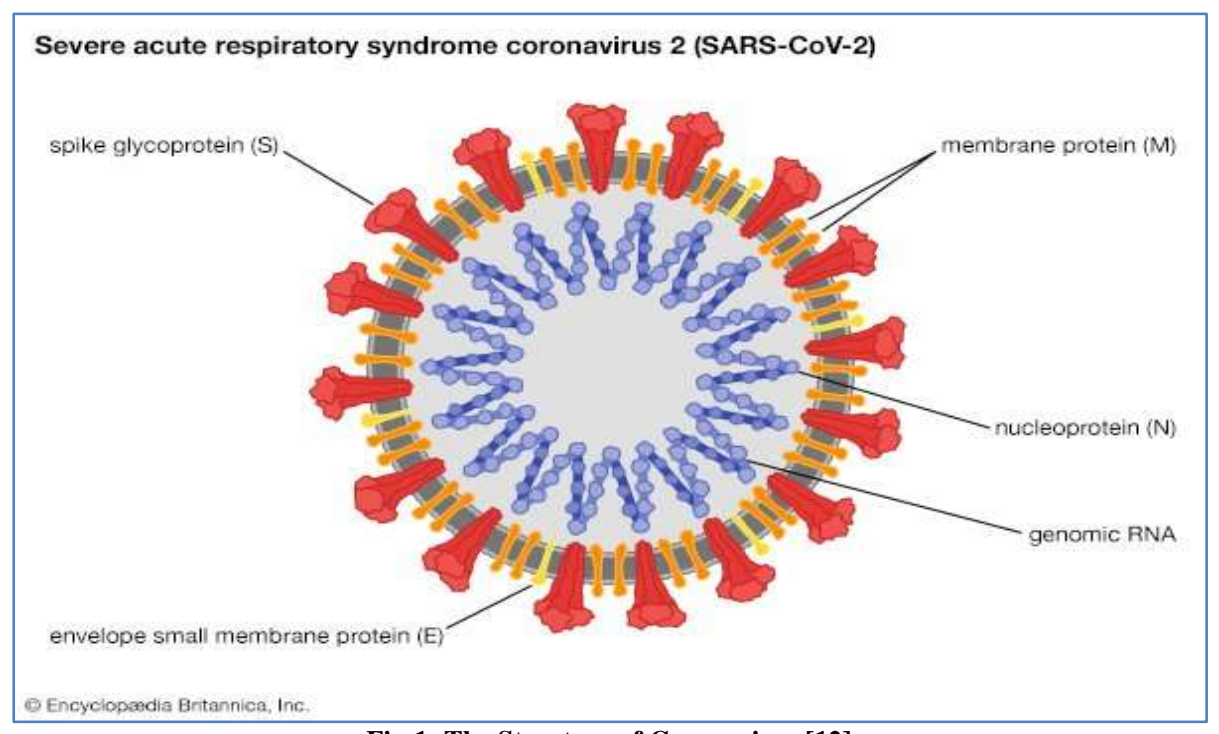

Fig-1: The Structure of Coronavirus [12]

Most people infected with the COVID-19 virus will experience mild to moderate respiratory illness and recover without requiring special treatment. Persons with COVID-19 present mostly with fever-like illness, a dry cough, dyspnoea, and bilateral infiltrates on chest imaging [7]. Other less severe symptoms include sore throat, aches and pains, headache, diarrhoea, conjunctivitis, a skin rash, the recent loss of taste or smell, or discolouration of fingers or toes [13].

The COVID-19 virus infects persons of all ages and genders, though infection in children has been observed to be less common and less severe than in adults because of their body system immunity. A systematic review reported that about $1-5 \%$ of children were infected with the COVID-19 virus. The children were mostly reported to have mild respiratory symptoms and they rarely died from the disease [14]. However, the risk of severe infection is higher among older people and those with underlying health challenges like diabetes mellitus, cardiovascular diseases, chronic respiratory diseases and cancers [15].
Of the 210 countries affected globally with COVID-19, Nigeria is one of such country. The first case was confirmed in Lagos State on 27 February 2020. This index case was a 44-year old man, an Italian citizen who returned from Milan, Italy, on 24 February and presented at a health facility on 26 February 2020 [16]. Following the confirmation of the index case, 216 people were identified as contacts to be followed up. Of these, 45 travelled out of Nigeria and one of the remaining 176 contacts was confirmed to be positive for COVID-19 on 9 March 2020 [17].

Some factors have been associated with the spread of the virus. Developing evidence proposes that cold and dry conditions appear to increase spread while humid and warm conditions may decrease the rate of infection [18-20]. High latitude has also been associated with higher rates of cases and deaths in some countries of the world [21]. Although, there is a need for additional studies to ascertain the validity of these discoveries. 


\section{Laboratory testing for COVID-19}

The best acceptable gold standard for testing methodology of COVID-19 is real time reverse transcription - polymerase chain reaction (RT-PCR) as recommended by WHO [10,22] and implemented by NCDC in country [10]. This method could be well positioned at the geopolitical centers while lesser and cheaper methodologies could go on at the state and local government public health laboratories thereby bringing the test closer to the people. This could be achieved with Rapid Detection and Point-of-Care Diagnostics for COVID-19 [10, 23]. The testing protocol is nevertheless dependent on the NCDC and WHO guidelines in the country (Nigeria).

In relation to China, NHC [22] and Nigeria [10] has showed certain standards that must be satisfied to be considered a potential COVID-19 for medical laboratory testing. They include: Epidemiological history and Clinical features of:

i. Symptoms of respiratory tract infection and fever,

ii. Radiographic evidence,

iii. Low or normal count of white-cell and lymphocyte.

Patients possessing an epidemiological history, consistent with any two clinical features, are diagnosed as suspected cases. COVID-19 suspected cases are tested for confirmation by:

a) Positive for SARS-COV-2 using respiratory tract or serum specimens which are examined with real-time RT-PCR;

b) The genome sequencing of the novel virus from respiratory tract or serum specimens which are identical to SARS-COV-2 sequences.

On the other hand, the quality of PCR results for COVID-19 is directly proportional to the quality of the kits and that of the medical laboratory scientist or the laboratorian doing the testing as well as to the novel coronavirus's features, sampling location, sampling volume, transportation and storage, coupled with laboratory test environmental conditions. The principle is based on the fact that COVID-19 interrelates with the angiotensin-converting enzyme2 (ACE-2) receptor, a cell-surface protein mainly found on lung alveolar epithelial cells [24]. The procedural methodology with principles of RT-PCR is as described by Wang et al., [25].

The alveolar lavage, sputum, pharynx swab are good samples for COVID-19 testing [15] nevertheless, pharyngeal swabs are the most common sampling method, because of unproductive coughs but may cause missed diagnoses sometimes in COVID-19 diagnosis. In addition, RT-PCR detection may be time consuming thereby increasing the testing turn-around time and inturn hamper the control of infectious diseases.
Therefore, the revised version of the 5th edition added clinical confirmed standards for the Hubei Province [26] and as such necessary for consideration in Nigeria. It is also, very important to consider the use of rapid test kit as preliminary screening before its confirmation with RT-PCR.

The NCDC planned for a Public Health Laboratory Network that shall cut across the states of the country (Federal Republic of Nigeria and Federal Capital Territory), targeting the year 2021, but the emergence of COVID-19, made NCDC to announce 2testing sites which has increased to 18 with 2 under construction as at 30th April 2020. It is instructive to note that at the time of writing this review, the laboratory sites according to geopolitical regions are South West - 7 Laboratories (Lagos, Ibadan, Ogun and Osun), South South - 1 Laboratory (Edo), South East -1 Laboratory (Ebonyi), North Central/ FCT - 3 Laboratory (Abuja, Jos,) North West -5 Laboratory (Sokoto, Kano and Kaduna) and North East - 1 Laboratory (Maiduguri). It is therefore very urgent for the Nigerian government through NCDC to plan towards more COVID-19 laboratories in all state capitals and supplementary one in very large states for adequate handling of the Nigerian populace [27].

For the diagnosis of COVID-19, the samples involved are blood, sputum, nasal secretions and bronchoalveolar lavage (BAL). The samples are usually subjected to specific serological and molecular tests specific for COVID-19 for laboratory diagnosis or testing. Serological tests employ Enzyme Linked Immunosorbent Assay (ELISA) or Western blots that detects specific COVID-19 proteins. Molecular approaches are based on Real Time-PCR (RT-PCR) or Northern blot hybridization with target of specific COVID-19 genes $[10,28]$. The viral antigens present in the clinical specimens can also detected by using direct immune fluorescent assay (IFA) [29,30] and Antigen/Antibody reaction testing [31].

With the continued shortage of testing centers, testing kits and PCR testing kits across the globe and mostly in Nigeria, there is need to consider rapid testing using new detection medical laboratory technology and point of care testing kits [32,33] or creating mobile biosafety laboratory as exemplified in Wuhan china [34] where the coronavirus started of recent. The NCDC have developed a very good safety and diagnosis procedures [10], however, the clarity in terms of job description and roles of the laboratory staff in the public health laboratories was not clearly stated. This calls for concern on the quality of test results expected in line with international best practice standards. There is a need to postulate the role of individuals involved in the diagnosis such as Pathologists, Medical Laboratory Scientists, Public and Environmental Health professionals and logisticians in the scheme of planned public health laboratories. 


\section{Precautionary measures with WHO/National Guidelines}

In line with WHO, the FMOH [35] and Obeta et al. [27] advised Nigerians for their good hand and respiratory hygiene with necessary precautions on personal, respiratory and Hand hygiene including; physical distancing (least 6 feet), avoidance of crowd (more than 20 people), adequate disposal of the infected materials or used tissue; avoidance of self-medication and contact of proper authority and staying informed through official channels of FMOH and NCDC. As a way to ensure adequate precautions and quality good medical laboratory practice standard during COVID-19 pandemic [10]. NCDC has provided some guides to Medical Laboratory Scientists and other health professionals working in COVID-19 testing centers. Coronavirus (COVID-19) Specimen Collection Guide deals with adequate labeling, Virus Transport Media (VTM) tube and any other sample tube standard packaging procedures as required by the NCDC with regards to COVID-19 sample site collection, adequate personal protection equipment (PPE) and hand hygiene.

\section{How is COVID-19 different from other coronaviruses?}

COVID-19 or SARS-COV-2 like the MERS and SARS coronaviruses was likely evolved from a virus previously found in animals. These viruses infects people of all ages and genders with evidence that older people and those with underlying medical conditions are at a higher risk of getting severe COVID-19 disease, unlike other coronaviruses that cause a significant percentage of colds in adults and children that are not a serious threat for healthy adults $[11,36]$. A good reason could be due to high viral loads of SARSCoV-2 that have been reported in the upper and lower respiratory tracts of patients comparing with SARS-CoV-1 hence the rate of spread of SARS-CoV-2 is higher than that of SARS-CoV-1 [37]. Also, due to the fact that no one has immunity to COVID-19, it means thousands to millions of people are likely to be more susceptible to viral infection and severe disease. It was reported by the WHO that about $3.4 \%$ of COVID-19 cases have died till the 3rd March 2020 [38]. In relation to the genomic structure, SARS-CoV-2 has an open reading frame (ORF3b) different from that of SARS-CoVs and a secreted protein that is ORF8 encoded [39]. Tang et al. [40] also reported that spike structure of SARS-CoV-2 has functional site variations in the receptor-binding domain compared to viruses from pangolin SARSrCoVs, which could be the resultant effects of mutations and natural selection, enhancing viral transmission and observable host debility. In fact, Angeletti et al. [41] stated that it is the mutational changes at positions 723 and 1010 involving the replacement of glycine amino acid with serine and isoleucine by proline respectively in the ORF1ab encoded 2 (nsp2) and nsp3.

\section{Management and Treatment of Coronavirus}

There is a management guideline by WHO globally as adopted by NCDC for use in Nigeria [10]. The Mainland Hospital in Yaba, Lagos State is the first hospital designated for the management of COVID-19 cases in Lagos and was the hospital that received and treated Nigeria's first cases of the disease. As Nigeria rises to the challenge of curtailing COVID-19 [42], achievement will largely depend on a comprehensive understanding of the history, symptoms and clinical profile of COVID-19 cases in our population and the case management approach. COVID-19 currently do not have a confirmed treatment nevertheless management is ongoing with various clinical trials with hope of developing/discovering vaccine as soon as possible. Many people have been treatment and reported negative in various countries including Nigeria. Several trials made so far includes: Japan flu drugs, antiviral drug - Favipiravir or Avigan, Chloroquine and hydroxychloroquine, failed Ebola drug - remdesivir, HIV drug combination- combination of lopinavir and ritonavir, An immunosuppressant and an arthritis drug - Actemra, or tocilizumab with interleukin 6 (IL-6) ie cytokine, sarilumab or tocilizumab, blood pressure drug- Losartan, and possible use of large dose of Vitamin C. [25,43]. Though, Zhi Young [43], started a clinical trial in Zhongnan Hospital, China from 11th February, 2020, with some countries looking the other way in the issue of Vitamin C infusion [44], idea presented by Ohanube and Obeta [45] on the use of Vitamin $\mathrm{C}$ for the treatment of COVID-19 could be another breakthrough.

The Presidential Task Force (PTF), NCDC and NAFDAC in collaboration with CDC and WHO should look into the use of indigenous products that could help in the management of COVID-19. Such products should include herbs such as Nem tree (Dogon yaro), and Nigerian food like vegetables and fruits - Lemon, Bitter cola, Ginger, Garlic and others.

\section{Is Vaccine available for COVID-19?}

There are at present no vaccines against coronaviruses, including COVID-19 or SARS-CoV-2. This is why it is very important to prevent infection or comprehend further spread of infection [46]. The development of vaccines is not an easy task because it takes time to be developed. Several pharmaceutical companies are working on vaccine candidates (different kinds of vaccines). It wills, nevertheless, take months or years before any vaccine can be broadly used as it needs to undergo extensive testing to determine its safety and efficacy [47].

\section{Ethical Implications of COVID-19}

Several ethical matters arise in any pandemic as observed during influenza pandemic just like COVID-19 presently ravaging the globe and Nigeria in 
particular. Such ethical anxieties are noticed during planning, organizing and responses to issues surrounding any pandemic and no wonder it is very important as future pandemics are important to consider, so as to ensure that response efforts are not delayed in the event of any pandemic. The precedence for access to healthcare by patients during COVID-19 pandemic in Nigeria is of great anxiety especially as Kano State citizens cry out due to neglect of treatment of the masses who need adequate healthcare and are been ignored because of the attention given to treating potential COVID-19 patients. Though such treatment might have been necessitated by limited of resources with increased demand of care and scarcity of human resources for health and personal protective equipment (PPE). The responsibilities of healthcare workers in amidst risks to their own health is a big problem; and the balance between reducing disease spread through isolation, and travel measures whilst protecting the right of individuals to freedom treatment and movement creates and ethical matters [48].

Nuffield council on Bioethics [49], emphasized that the public health measures need to be evidence-based and proportionate to the public. It is therefore imperative to add that intimidation and intrusion into people's lives during the COVID-19 should be as minimum as possible and consistent with treating all with respect, morality and equality. While public individuals in Nigeria are asked by the government to make sacrifices for the public good, the respect and fundamental human rights due for individuals should never be forgotten in the way in which interventions such as quarantine and selfisolation are implemented at any giving period. Prominently, solidarity is a crucial part of COVID-19 intervention at the international level between countries like China and Nigeria for instance; private and business personalities in exercising their corporate social responsibility; and at the individual level in the way we all respond to the outbreak in day-to-day life of various people without discrimination and stigmatization. The ethical matters of informed consent, confidentiality and right of movement is challenged by COVID-19 in Nigeria, nevertheless, what is right and acceptable shall be applied to ensure safety and containment of the COVID-19 pandemic in this country.

\section{CONCLUSION}

This review greatly summarised some important basic facts about COVID-19 or SARS-COV2 pandemic in Nigeria as it continued to ravage the whole world. The better understanding of the COVID19 basic facts such as, origin, spread, morphology, symptom, diagnosis, precautionary measures, management and treatment, vaccine design and development, pathogenicity and ethical implications that is still limited will assist in founding best ways of fighting the pandemic. Also, every care must be taken to minimised human to human transmission of the virus by adherence to recommendations and guidelines on COVID-19 by FMOH/NCDC/FG. Further research and better understanding of the virus will help in the design and development of vaccines and better medications against the virus.

\section{Recommendations for FMOH/NCDC/FG on COVID-19}

i. Continue the construction of State by State laboratory network for easy access and quality medical laboratory testing (diagnosis) for all.

ii. Decentralize testing of COVID-19 and possibly use regional centers as confirmation where the states cannot confirm.

iii. Encourage local production of COVID-19 materials and consumables such as face masks, goggles, shoes, sanitizers, clinical and laboratory wears, COVID-19 test kits and COVID-19 transport medium (Virus transport medium-VTM).

\section{REFERENCES}

1. Tyrrell, D. A. J., Almeida, J. D., Cunningham, C. H., Dowdle, W. R., Hofstad, M. S., McIntosh, K., .. \& Bingham, R. W. (1975). Coronaviridae. Intervirology, 5(1-2), 76.

2. Yin, Y., \& Wunderink, R. G. (2018). MERS, SARS and other coronaviruses as causes of pneumonia. Respirology, 23(2), 130-137.

3. World Health Organization (WHO). (2020). SARS (Severe Acute Respiratory Syndrome) Geneva [4 May 2020]. Available from: https://www.who.int/ith/diseases/sars/en /.

4. MMWR. (2013). Updated Information on the Epidemiology of Middle East Respiratory Syndrome Coronavirus (MERS-CoV) Infection and Guidance for the Public, Clinicians, and Public Health Authorities, 2012-2013. Morbidity and Mortality Weekly Report 2013/V; 62: 793-796.

5. Zhu, N., Zhang, D., Wang, W., Li, X., Yang, B., \& Song, J. (2019). A novel Coronavirus from patients with pneumonia in China, N Engl J Med. Published Online First: 24 January 2020. doi: https://doi.org/10.1056/NEJMoa2001017.

6. China Daily Statistics. (2020). Daily Reports statistics COVID-19.

7. Chan, J.F-W., Yuan, S., Kok, K-H., To, KK-W., Chu, H., \& Yang, J. (2020). A familial cluster of pneumonia associated with the 2019 novel Coronavirus indicating person-to-person transmission: a study of a family cluster. Lancet. Published Online First: 24 January 2020. doi: https://doi.org/10.1016/S01406736(20)30154-9.

8. Lu, R., Zhao, X., Li, J., Niu, P., Yang, B. \& Wu, H. (2020). Genomic characterisation and epidemiology of 2019 novel Coronavirus: implications for virus origins and receptor binding. Lancet. Published Online First: 30 January 2020. 
doi: $\quad$ https://doi.org/10.1016/ S01406736(20)30251-8.

9. Zhou, P., Yang, X. L., Wang, X. G., Hu, B., Zhang, L., Zhang, W. \& Chen, H. D. (2020). A pneumonia outbreak associated with a new coronavirus of probable bat origin. Nature, 579(7798), 270-273.

10. World Health Organization. (WHO). (2020). WHO Director-general's opening remarks at the media briefing on COVID-19: 11 March 2020. Published March 11, 2020. Accessed April 11, 2020. https://www.who.int/dg/speeches/detail/ whodirectorgeneral-s-opening-remarks-atthe-mediabriefingon-covid-19---11-April2020.

11. World Health Organization (WHO). (2020). Novel Coronavirus (2019-nCoV) Situation Report 1.2020. [Cited 14th April 2020].

12. Yang, Y., Lu, Q., Liu, M., Wang, Y., Zhang, A., Jalali, N., \& Zhang, X. (2020). Epidemiological and clinical features of the 2019 novel coronavirus outbreak in China. MedRxiv.

13. Phan, L. T., Nguyen, T. V., Luong, Q. C., Nguyen, T. V., Nguyen, H. T., Le, H. Q., ... \& Pham, Q. D. (2020). Importation and human-to-human transmission of a novel coronavirus in Vietnam. New England Journal of Medicine, 382(9), 872-874.

14. Ludvigsson, J.F. (2020). Systematic review of COVID19 in children shows milder cases and a better prognosis than adults. Acta Paediatr. [Epub ahead of print].

15. Huang, C., Wang, Y., Li, X., Ren, L., Zhao, J. \& $\mathrm{Hu}, \mathrm{Y}$. (2020). Clinical features of patients infected with 2019 novel Coronavirus in Wuhan, China. Lancet. Published Online First: 24 January 2020. doi: https://doi.org/10.1016/S01406736(20)301835.

16. Nigeria Centre for Disease Control. (2020). First Case of Corona virus Disease Confirmed in Nigeria [Internet]. 2020 [cited 2020 Apr 1]. Available from: https://ncdc.gov.ng/news/227/first-caseofcorona-virus-disease-confirmed-in-nigeria.

17. Nigeria Centre for Disease Control. (2020). COVID-19 Outbreak in Nigeria Situation Report S/N 54. Abuja; 2020. [Internet]. 2020 [cited 2020 Apr 23]. Available from: https://ncdc.gov.ng/diseases/sitreps/?cat=14\& name $=$ An $\% 20$ update $\% 20$ of $\% 20$ COVID $19 \% 20$ out break\%20in\%20Nigeria. 7

18. Centre for Evidence-Based Medicine (CEBM). (2020). Brassey J, Heneghan C, Mahtani KR, Aronson K. Do weather conditions influence the transmission of the Coronavirus (SARSCoV-2)? 2020 [internet publication].

19. Ujiie, M., Tsuzuki, S., \& Ohmagari, N. (2020). Effect of temperature on the infectivity of COVID19. Int J Infect Dis 2020 [Epub ahead of print].

20. Shi, P., Dong, Y., Yan, H., Zhao, C., Li, X., \& He, M. (2020). Impact of temperature on the dynamics of the COVID-19 outbreak in China. Sci Total Environ, 728: 138890.
21. Centre for Evidence-Based Medicine; Heneghan, C., Jefferson, T. (2020). Effect of latitude on COVID-19. [Internet Publication].

22. Yu, H., \& Hailan, Yang. (2020). The transmission and diagnosis of 2019 novel coronavirus infection disease (COVID-19): A Chinese perspective. Journal of Medical Virology.

23. Nassiri, N. (2020). Perspective on Wuhan Viral Pneumonia. Adv in Pub Health, Com and Trop Med: APCTM-106. Kosmos Publishers.

24. Hamming, I., Timens, W., Bulthuis, M.L., Lely, A.T. \& Navis, G. (2004). Tissue distribution of ACE2 protein, the functional receptor for SARS coronavirus. A first step in understanding SARS pathogenesis. J Pathol, 203: 631-637.

25. Wang, M., Cao, R., Zhang, L., Xinglou, Y. \& Jia, L. (2020). Remdesivir and chloroquine effectively inhibit the recently emerged novel coronavirus (2019-nCoV) in vitro. Cell Res 30: 269-271.45.

26. National Health Commission of the People's Republic of China (NHCPRC). The notice of launching guideline on diagnosis and treatment of the novel coronavirus pneumonia (NCP). Revised version of the (5th edn).

27. Obeta, M.U., Ejinaka, R.O., Ofor, I.B., Ikeagwulonu, R.C. \& Agbo, E.C. (2020). Nigerian COVID-19 (Coronavirus) Patients Update, the Realities with Medical Laboratory Diagnostic Sites. American Journal of Epidemiology and Infectious Disease 8(1): 13-15.

28. Kannan, S.P.A.S., Shaik, A., \& Sheeza, Hemalatha, K. (2020). COVID-19 (Novel Coronavirus 2019) recent trends. European Review for Medical and Pharmacological Sciences. 24: 2006-2011.

29. Bruning, A.H., Aatola, H., Toivola, H., Ikonen, N. \& Savolainen, K.C. (2018). Rapid detection and monitoring of human coronavirus infections. New Microbes New Infect 24: 52-55.

30. Corman, V.M., Landt, O., Kaiser, M., Molenkamp, R. \& Meijer, A. (2020). Detection of 2019 novel coronavirus (2019-nCoV) by real-time RT-PCR. Eurosurveillance 25: 2000045.

31. Chu, D.K.W., Pan, Y., Cheng, S.M.S., Hui, K.P.Y. \& Krishnan, P. (2020). Molecular Diagnosis of a Novel Coronavirus (2019-nCoV) Causing an Outbreak of Pneumonia. Clin Chem, 7: 549-555.

32. Trieu, N., Dang Duong, B., \& Anders, Wolff. (2010). 2019 Novel Coronavirus Disease (COVID19): Paving the Road for Rapid Detection and Point-of-Care Diagnostics Micromachines 11: 306.

33. BGI. (2020). New Emergency Detection Laboratory Run by BGI Starts Trial Operation in Wuhan, Designed to Test 10,000 Samples Daily. Assessed on 23rd March 2020.

34. CCDC. (2020.) Assistance to Wuhan, High-level Mobile Biosafety Lab Departs. Accessed on 23rd March 2020.

35. FMOH. (2020). First case of Coronavirus disease confirmed in Nigeria. (2020) Pneumonia of 
unknown cause - China. World Health Organization.

36. OSHA. (2020). COVID-19. Occupational safety and health administration. [Online]. Available from: https://www.osha.gov/SLTC/covid-19/ medicalinformation.html. [Cited on 13 March 2020].

37. Promed. (2020). Latest on COVID-19: International society for infectious diseases. [Online]. Available from: https://promedmail.org/. [Cited on 15 March 2020].

38. $\mathrm{WHO}_{\mathrm{e}}$. (2020). "We now have a name for the \#2019nCoV disease: COVID-19. I 'll spell it: COV-I-D hyphen one nine - COVID-19", Tweet, 11 February, viewed 11 February 2020, https://twitter.com/WHO/status/1227248333 871173632

39. Cascella, M., Rajnik, M., \& Cuomo A. (2020). Features, evaluation and treatment coronavirus (COVID-19). Treasure Island (FL): StatPearls Publishing; 2020.

40. Tang, X., Wu, C., Li, X., Song, Y., Yao, X., \& Wu, $X$. (2020). On the origin and continuing evolution of SARS-CoV-2. National Sci Rev 2020. doi: https:// doi.org/10.1093/nsr/nwaa036.

41. Angeletti, S., Benvenuto, D., Bianchi, M., Giovanetti, M., Pascarella, S., \& Ciccozzi, M. (2020). COVID-2019: The role of the nsp2 and nsp3 in its pathogenesis. J Med Virol, 2020. doi: $10.1002 / j m v .25719$.
42. Nigeria Center for Disease Control. (2020). COVID-19 Nigeria. 2020. Accessed April 7, 2020.

43. Zhi, Y.P. (2020). Vitamin C Infusion for the Treatment of Severe 2019-nCoV Infected Pneumonia.

44. DoH Australia. (2020). No evidence to support intravenous high-dose vitamin $\mathrm{C}$ in the management of COVID-19. Alert/Advisory, Medicines safety. Accessed on 25th March 2010.

45. Ohanube, G.A.K., \& Obeta, M.U. (2020). COVID19: Novel Opinion on Strategic Prophylaxis and Cure Using Vitamin C (Ascorbic Acid). Acta Scientific Nutritional Health, 4(5): 32-33.

46. Guangxi University Report. (2020). Daily Report of COVID-19 dated 23 February 2020 - China. Holmes, E. (2020). Initial genome release of novel coronavirus 2020 [14 January, 2020]. Available from: http://virological.org/t/initial-genome-rel ease-of-nov elcoronavirus/319.

47. European Centre for Disease Prevention and Control (ECDPC). (2020). Risk assessment: Outbreak of acute respiratory syndrome associated with a novel coronavirus,

48. WHO. (2020). Ethical issues in pandemic influenza planning?

49. NCB. (2020). New briefing: Responding to the COVID-19 pandemic - ethical considerations.50. NCDC (2020) National Laboratory Network. 